Service social

\title{
La Société canadienne du sida
}

\section{Richard Burzinski}

Volume 38, numéro 1, 1989

Aspects psychosociaux du Sida

URI : https://id.erudit.org/iderudit/706420ar

DOI : https://doi.org/10.7202/706420ar

Aller au sommaire du numéro

Éditeur(s)

École de service social de l'Université Laval

ISSN

1708-1734 (numérique)

Découvrir la revue

Citer cet article

Burzinski, R. (1989). La Société canadienne du sida. Service social, 38(1), 17-21. https://doi.org/10.7202/706420ar

\section{Résumé de l'article}

Cette Société regroupe, à l'échelle canadienne, 39 organismes communautaires dont les objectifs ont trait à la prévention de l'infection VIH et à l'assistance des personnes touchées par le sida. Par ses activités, elle vise à aider les

organismes membres à se maintenir à l'avant-garde du développement et de la dispensation de services de prévention et d'assistance dans leur communauté.

La perspective de base des programmes communautaires sur le sida est de voir cette maladie comme un problème collectif. d'utilisation que vous pouvez consulter en ligne.

https://apropos.erudit.org/fr/usagers/politique-dutilisation/ 


\section{La Société canadienne du sida *}

\section{Richard Burzinski}

La Société canadienne du sida, qui a son siège social à Ottawa, a été créée en 1985 pour répondre aux besoins croissants des organismes communautaires impliqués dans des activités d'éducation ou dispensant des services directs. Elle regroupe trente-neuf organismes communautaires allant de Saint-Jean (Terre-Neuve) à Victoria. Au plan canadien, elle représente donc la réponse des diverses communautés au phénomène du sida.

\section{Organismes membres}

Les membres de cette Société sont des organismes communautaires sans but lucratif dont les objectifs principaux ont trait à la prévention de l'infection VIH et à l'assistance des personnes touchées par le sida et par les conditions de vie qui y sont liées. Un organisme peut devenir membre si au moins les deux tiers des délégués d'une assemblée régulière de la Société adoptent sa demande d'adhésion.

\section{Soutien aux organismes membres}

La Société canadienne du sida vise à aider ses organismes membres à se maintenir à l'avant-garde du développement et de la dispensation de services de prévention et d'assistance dans leur communauté. Elle sert ses membres de deux façons distinctes mais interreliées, soit par des programmes internes à la Société ou par des programmes principalement dispensés à l'extérieur de celle-ci. Ces deux domaines d'activités forment ensemble, pour le Canada, la réponse communautaire de lutte contre le sida. 
Les activités internes de soutien aux membres de la Société incluent les communications, l'élaboration de programmes, l'aide au développement de ressources et la création de réseaux communautaires. Parmi les activités externes dont bénéficient les membres, notons les relations et les projets conjoints avec d'autres organismes nationaux, la liaison avec les divers paliers de gouvernement et avec les bailleurs de fonds, ainsi que le parrainage de rencontres et de symposiums à l'échelle nationale.

\section{Services publics et services communautaires}

Malgré une lente augmentation du budget fédéral alloué à la problématique du sida, la plus grande partie de ces fonds est utilisée pour l'information de masse, l'éducation et la recherche.

Compte tenu de l'ampleur de la crise du sida et de la lenteur de la réaction initiale du gouvernement fédéral, des organismes se sont développés au plan local. En conséquence, ceux-ci ont pris le leadership dans les communautés les plus touchées de façon à faire face à cette maladie et à fournir les services essentiels aux personnes qui en souffrent.

\section{Services offerts}

Écoute téléphonique. Il s'agit souvent ici de la dispensation d'informations orientées vers des groupes de personnes spécifiques, par exemple la communauté gaie, les jeunes, des personnes voulant obtenir des traitements à l'état expérimental, ou d'autres voulant être référées à des avocats, dentistes ou médecins.

Ressources d'accueil. Par exemple, la Maison Casey, à Toronto, a été fondée à l'intention des sidéens en phase terminale.

Aide par les pairs. Des bénévoles fournissent accompagnement, consultation, défense des droits et assistance dans des tâches quotidiennes comme la préparation des repas, le ménage, le marché et les transports.

Programmes éducatifs. Des professionnels et des bénévoles élaborent des messages éducatifs appropriés qui ont pour cible, par exemple, la communauté gaie ou les étudiants, ou qui s'adressent à la population en général. Ils mettent sur pied des ateliers à l'intention de professeurs dans leur propre milieu. De tels programmes peuvent inclure des ateliers sur le safer-sex et des messages éducatifs utilisant la bande dessinée, à l'intention des itinérants ou d'une population illettrée. 
Les autres services développés dans la communauté comprennent : des services de counseling et des groupes de support pour les personnes séropositives ou atteintes du sida; des services juridiques pour la rédaction de testaments et la lutte contre la discrimination. De nombreux programmes déjà développés dans des organismes communautaires à l'intention de la population gaie servent de modèles aux groupes qui commencent à fournir des services semblables à d'autres populations.

\section{Les bénévoles et leur implication}

Les réponses initiées par les communautés se servent aussi de la longue tradition canadienne de bénévolat. De nombreux services ont été fondés par des hommes et des femmes sensibilisés au tragique de la maladie et mûs par le sens des responsabilités, et qui se portent volontaires pour de nombreuses heures de bénévolat. Des personnes de toutes les sphères de la société ont fourni leur contribution, apportant du réconfort et une aide parfois inestimable. En 1988, plus de 3000 Canadiens ont donné de leur temps et de leurs énergies aux organismes membres de la Société canadienne du sida, consacrant ainsi plus de 240000 heures à la lutte contre cette maladie.

Les personnes elles-mêmes touchées joignent souvent les rangs des bénévoles. Puisque le sida se différencie des autres maladies graves par le degré variable de diminution des capacités chez les individus qui en souffrent, plusieurs peuvent ainsi continuer une vie active, conservant leur emploi et fonctionnant comme ils le faisaient avant la confirmation de la maladie. En conséquence, ils s'impliquent souvent dans les organismes communautaires - comme intervenants, bénévoles ou membres du conseil d'administration - et fournissent ainsi une ressource dont les concepteurs de programmes et services pour sidéens peuvent difficilement évaluer l'impact.

Cette implication du " patient " ou du « client " dans le développement des services constitue une autre façon par laquelle les organismes communautaires se différencient du modèle médical traditionnel où aidant et aidé ont chacun un rôle défini : l'aidant bien portant fournit des services à l'aidé malade.

\section{Le travail d'éducation}

Une autre contribution essentielle des organismes communautaires est l'éducation très localisée et ciblée afin d'infléchir la croissance du $\mathrm{VIH}$. Le financement provenant du gouvernement fédéral supporte aussi maintenant l'éducation du public en général sur la problématique 
du sida, ce qui constitue un point important pour créer un climat favorable et rendre les individus plus réceptifs aux messages préventifs.

Les programmes communautaires en sont rendus à l'étape cruciale d'élaborer des messages éducatifs qui tiennent compte des besoins et des attitudes de communautés spécifiques. En utilisant un langage et des images explicites, ils veulent rejoindre des individus ayant des comportements à risque tels que les contacts sexuels non protégés et l'usage d'aiguilles contaminées. Ils élaborent aussi des messages spécifiques qui tiennent compte des barrières socio-économiques et qui permettent de rejoindre des individus différents par leur ethnicité, leur culture ou leur comportement sexuel.

Parmi les programmes innovateurs d'éducation communautaire ayant pour cible des individus typiques par rapport à la population en général, le projet " Prostitute's Safer Sex ", à Toronto, utilise des personnes qui font le commerce du sexe pour informer leurs collègues des méthodes permettant de réduire les risques.

\section{Les aspects collectifs}

Par l'entremise de ses membres, la Société canadienne du sida prête main forte à tous les Canadiens touchés par cette maladie. Elle milite, par exemple, pour que les sidéens et les personnes séropositives aient plus facilement accès aux médicaments encore à l'état expérimental. Alors que les Américains peuvent bénéficier de plus de quarante médicaments pour le traitement du sida, les Canadiens doivent se satisfaire de quatre seulement parmi ceux-ci. La Société travaille aussi à l'obtention de subventions gouvernementales plus élevées afin de lutter plus efficacement contre ce fléau. Particulièrement, elle tente d'obtenir des fonds pour les organismes membres dont les ressources sont insuffisantes mais qui, d'autre part, s'appuient sur la générosité de leur collectivité. De plus, elle sert de réseau d'échange pour ses membres, leur permettant de partager connaissances, expériences, informations et problèmes.

\section{Conclusion}

Un modèle de services relatifs au sida qui a une orientation communautaire et une perspective de continuité de soins entraîne une réduction significative des coûts de traitement et une qualité de vie supérieure pour les sidéens; en effet, une bonne partie des services sont alors offerts à l'extérieur de l'hôpital par des réseaux de soins 
médicaux et de programmes psychosociaux où œuvrent, pour une bonne part, des bénévoles.

La perspective de base des programmes communautaires sur le sida est de voir cette maladie comme un problème collectif. Si une communauté veut se concerter et aider ses membres, des changements importants peuvent se produire. Ainsi, il y a réduction des coûts; la compassion n'est plus un simple concept ; la récompense des membres vient de la gratification provoquée par le sentiment d'aider d'autres individus dans la communauté. La dignité des personnes sidéennes est respectée si l'on s'adresse à elles comme à des individus ayant les mêmes besoins et désirs que quiconque. De cette façon, le travail s'accomplit, on satisfait les besoins humains et tous en bénéficient.

\section{Note}

* Traduit et adapté par Jocelyn Lindsay. 